\title{
GAMING COMO MÉTODO DE INTERVENCIÓN EN EL TRASTORNO POR DÉFICIT DE ATENCIÓN E HIPERACTIVIDAD
}

\author{
GAMING AS AN INTERVENTION METHOD IN ATTENTION DEFICIT \\ HYPERACTIVITY DISORDER
}

\section{Jorge Casado García', José María Villa Andrada ${ }^{2}$}

\begin{abstract}
RESUMEN
El presente estudio tiene como objetivo realizar una intervención utilizando como base los videojuegos. Para el mismo, se ha utilizado un videojuego indie llamado GRIS. El estudio es de tipo cuasi-experimental, donde se ha evaluado a los niños/as antes y después de la intervención. La evaluación de la intervención se realizó ha realizado mediante el test de Toulouse, que se mide mediante el índice global de atención y percepción (IGAP), y una guía creada específicamente para este estudio. El grupo de participantes, seleccionado por conveniencia, se conformó por tres niños procedentes del centro DIVERTEA en Cáceres (España) que presentaban trastorno por déficit de atención e hiperactividad o déficit de atención como trastorno comórbido. La comparación de los resultados obtenidos en los test previo y posterior indican una mejora en la atención y en la percepción, con unos resultados entre el $80 \%$ y el 1oo por ciento en el IGAP posterior a la intervención. Estos resultados refuerzan la idea de utilizar los videojuegos como método de intervención para el trastorno por déficit de atención e hiperactividad. Queda abierta la posibilidad de seguir investigando sobre la utilización de los videojuegos como método de intervención en otros tipos de patologías y trastornos.
\end{abstract}

\section{PALABRAS CLAVE}

Juegos de Video, Atención, Trastorno por Déficit de Atención con Hiperactividad, Terapia Ocupacional

1 Graduado en Terapia Ocupacional por la Universidad de Extremadura. Correspondencia: C/General Margallo $1102^{\circ} \mathrm{A}, \mathrm{CP} 10003$, Cáceres (Cáceres) jorgecasado1997@gmail.com Teléfono: 645402363 ORCHID: 0000-0001-8075-8580

2 Diplomado en Enfermería por la Universidad de Extremadura. Titulado Superior en Enfermería por la Universidad de Alicante. Master Oficial en Ciencias de la Enfermería por la Universidad de Alicante. Master en Dirección de Organizaciones Sanitarias y Sociosanitarias por la Universidad de Extremadura. Coordinador del Punto de Atención Continuada de Cáceres. Servicio Extremeño de Salud. Extremadura. España. Profesor Asociado. Facultad de Enfermería y Terapia Ocupacional de Cáceres. Universidad de Extremadura. España. Correspondencia: Facultad de Enfermería y Terapia Ocupacional. Avd. de la Universidad s/n. CP 10003, Cáceres (Cáceres) jmvillaa@unex.es Teléfono: 626149601. ORCID: 0000-0003-4012-9733 


\begin{abstract}
The aim of this study was to deliver an intervention using videogames as a base. For this study, it was used an indie videogame called GRIS. This was a quasi-experimental study, where children were evaluated before and after the intervention. For the assessment, Toulouse test was used, which is measured by global index of attention and perception (GIAP) and a guide created specifically for this study. The group of participants, selected by convenience, was made up of three children from DIVERTEA in Caceres (Spain) which had attention deficit/hyperactivity disorder, or attention deficit as a co-morbid disorder. The results obtained with the pre.post testing were compared, suggesting an attention and perception improvement with result between 80 and 100 percent in GIAP post-intervention. This result reinforces the idea of using videogames as intervention method in attention deficit/hyperactivity disorder. The possibility of using videogames as an intervention method in other pathologies and disorders, is open to continue research.
\end{abstract}

\title{
KEYWORDS
}

Video Games, Attention, Attention Deficit/Hyperactivity Disorder, Occupational Therapy

Recibido: 09/03/2020

Aceptado: 05/09/2020

\section{INTRODUCCIÓN}

El Trastorno por Déficit de Atención e Hiperactividad (TDAH) es un trastorno neurobiológico frecuente en la infancia, asociado a un importante impacto funcional, personal y social. Presenta un gran componente genético, con múltiples genes implicados que, a su vez, interactúan con factores ambientales y neurobiológicos (Rodillo, 2015). El TDAH se define como un patrón persistente de inatención, hiperactividad-impulsividad que interfieren en el funcionamiento o en el desarrollo de la persona.

La inatención se caracteriza por dificultades al prestar atención a los detalles, tareas o actividades cometiendo errores en las mismas. Además, parece no escuchar cuando se le habla directamente, se distrae con facilidad por estímulos externos y presenta dificultades para seguir instrucciones y organizar tareas. La hiperactividad e impulsividad se caracteriza por una incapaz para permanecer quieto en un sitio sin levantarse, hacer ruidos o juegos con sus manos, además, de hablar excesivamente, respondiendo antes de que haya concluido la pregunta e interrumpiendo con frecuencia (American Psychiatric Association, 2013).

Estos síntomas están presentes antes de los 7 años y suelen presentarse en dos o más lugares diferentes, como pueden ser en casa y en el colegio. Presentan una afec- • tación a nivel académico, social y ocupacional, sin que tales síntomas sean debidos a algún otro tipo de trastorno (Sánchez, Sánchez, \& Suárez, 2015).

Este trastorno es crónico y requiere de un tratamiento a largo plazo, con el correspondiente coste social (Pérez, 2015). Cabe destacar que no existe un tratamiento curativo, de forma que el objetivo principal es disminuir la intensidad y la frecuencia de sus síntomas, mejorando así la calidad de vida del paciente. Este tratamiento debe ser multimodal, formado por tres tipos de tratamientos (Fontiveros et al., 2015):

- Farmacológico: se prescribe un tratamiento farmacológico en el que la elección de los fármacos depende del paciente, de sus comorbilidades, edad y preferencias, además de las propias características del fármaco (Fontiveros et al., 2015).

- Psicológico: encontramos dos tipos de tratamientos psicológicos. Por un lado, encontramos la psicoeducación, que consiste en enseñar al paciente vías para afrontar problemas emocionales, conductuales y cognitivos asociados al trastorno, además de detectar posibles dificultades y definir sus propios objetivos terapéuticos. Por otro lado, se encuentra el tratamiento conductual, considerado el tratamiento no farmacológico más eficaz, ayudando a reducir la aparición de trastornos comórbidos o a disminuir la intensidad de los síntomas (Fontiveros et al., 2015).

Psicopedagógico: va dirigido al entrenamiento neuropsicológico en diferentes funciones del 
sistema ejecutivo, organización, flexibilidad cognitiva, motivación, memoria de trabajo... además de ajustes necesarios durante las clases y evaluaciones (Fontiveros et al., 2015).

No se conoce con exactitud que causa el TDAH, aunque se ha observado que gran parte de las causas son provocadas por factores genéticos, ambientales y psicosociales. Según el estudio de Rodríguez Molinero et al. (2009) se estimó que casi el $76 \%$ de las causas se podrían atribuir a los factores genéticos. Además, respecto a los factores ambientales, se realizó una asociación donde los principales de riesgo aparecen en el embarazo, el parto y después del parto. Por último, los factores psicosociales no son determinantes, pero adquieren un papel importante en el trastorno, sobre todo a la hora de realizar diversas intervenciones.

Los niños diagnosticados de TDAH suelen presentar un déficit en las funciones ejecutivas, tales como la inhibición del comportamiento, flexibilidad cognitiva, planificación y organización. Este déficit provoca un bajo rendimiento en la realización de tareas, lo que puede desembocar en dificultades en los ámbitos escolar, familiar y social del niño. (Rubiales, Bakker, Russo, \& González, 2016).

Los principales trastornos comórbidos relacionados con el TDAH son:

- Trastorno oposicional o negativista desafiante: caracterizado por un patrón frecuente y persistente del enfado o de irritabilidad, un comportamiento discutidor/desafiante o con deseo de venganza (Pérez, 2015).

- Trastorno de conducta: existe un continuo con el trastorno oposicional o negativista desafiante. Este trastorno se encuentra caracterizado por síntomas como agresiones, crueldad, robos, faltas a la escuela, mentiras, etc. (Zúñiga \& Forteza, 2014).

- Trastornos de humor o trastornos afectivos: caracterizado por síntomas depresivos con sentimientos de culpa, falta de autoestima, estado de ánimo irritable, falta de energía y somatizaciones. También pueden llegar a presentar otros tipos de trastornos como pueden ser el trastorno bipolar o distímico (Artigas-Pallarés, Nonell, \& Ratera, 2013).

- Trastorno del sueño: caracterizado por problemas para dormir, con una disminución de la necesidad de dormir (Artigas-Pallarés et al., 2013).
- Trastorno del aprendizaje: es uno de los trastornos comórbidos más frecuente. Está caracterizado por problemas de lectura o dislexia, con problemas en la escritura o disgrafía y alteraciones del cálculo matemático o discalculia (del Río, 2014).

- Ansiedad por separación: provoca un sufrimiento excesivo cuando se le separa de una persona que presenta un vínculo estrecho. Esto genera en la persona preocupación, malestar psicológico subjetivo y un rechazo a quedarse solo en casa o a desplazarse a otros lugares (American Psychiatric Association, 2013).

A su vez puede desencadenar otros trastornos, que no son tan frecuentes, como pueden ser el síndrome de la Tourette, trastornos obsesivos compulsivos, desórdenes en la comunicación y trastornos de la coordinación (Zúñiga \& Forteza, 2014).

Este estudio utiliza el gaming y los videojuegos como método de intervención y según el diccionario de Cambridge, gaming es "la actividad de jugar a videojuegos" (Cambrige Dictionary, 2017). Los videojuegos son una simulación digital sonora y de imágenes que son ejecutados en un dispositivo electrónico, como un ordenador, un teléfono móvil o una consola, con el fin de proveer entretenimiento y en función de la plataforma, el videojuego poseerá diferentes tipos de controles (Taniel \& Samuel, 2015). Un control de videojuego es un periférico de entrada que permite interactuar con un videojuego. Este periférico se sujeta con las dos manos y los pulgares se utilizan para la entrada de datos. Suelen tener tres tipos de botones: de acción, de dirección y de control (Rosa Cortés, 2015).

Para conocer el porcentaje de población, la edad y otros datos relevantes sobre el uso de los videojuegos, a comienzos del año 2018, se realizó una encuesta online, creada por la Interactive Software Federation of Europe (ISFE) (GameTrack digest: Quarter 3.2018).

Primero se evaluó de manera general el porcentaje de población que juega a videojuegos, donde en algunos países como Francia, supone el $64 \%$ de la población, un total de 7.3 horas a la semana. Por su parte, en España, el $45 \%$ de la población juega a videojuegos, un total de 5.5 horas a la semana. Estas horas fueron tomadas en un rango de edad de entre 11 y 64 años (GameTrack digest: Quarter 3.2018). 
En la actualidad, los videojuegos presentan dos puntos de vista completamente diferentes. Por un lado, encontramos un punto de vista negativo, que apunta a que los videojuegos pueden aumentar la posibilidad de crear en las personas impulsos agresivos o adicciones. Una de las hipótesis actuales es que el uso de videojuegos violentos puede provocar, mediante observación o realización de actos violentos, comportamientos que se generalicen al mundo real, con una posible insensibilización a sus efectos (Gómez, 2017).

Otro de los efectos negativos que presentan los videojuegos es su capacidad de adicción a los mismos, llegando a producir una falsa sensación de que el mundo es más real dentro de los videojuegos que fuera de ellos (Gómez, 2017).

El mal uso de los videojuegos y de las tecnologías en general puede generar otros efectos negativos, como puede ser una afectación en el rendimiento académico. En el estudio de Martín (2015) se analizó la relación entre el tiempo dedicado a la televisión y el uso de videojuegos con las notas de los alumnos en la escuela, y se llegó a la conclusión de que un mayor número de horas de televisión y videojuegos puede provocar un bajo rendimiento en la escuela.

Por otro lado, encontramos un punto de vista positivo, ya que existen gran variedad de estudios que analizan los principales beneficios que pueden llegar a generar (de Prado, 2018):

- Favorecen el desarrollo de inteligencias múltiples, competencias, capacidades y habilidades (sociales, metacognitivas, autonomía y competencia digital).

- Propuesta y experiencia.

- Motivación.

- Inclusión educativa y atención a la diversidad.

- Actitud favorable de los jóvenes y futuros docentes.

En la actualidad, se plantea la posibilidad de educar con videojuegos, donde el educador debe estar "en sintonía" con el público que quiere educary los videojuegos están "en sintonía" con los adolescentes actuales. A los niños/as actuales no les gusta leer y exigen interactividad, es por ello que esta educación debe producirse de manera digital a través de experiencias visuales, que a su vez se encuentren bien diseñadas (Valderrama-Ramos, 2011).
Respecto a la utilización de videojuegos como método de intervención para patologías y trastornos, la mayoría de los estudios que encontramos en las principales bases de datos como Dialnet o Medline utilizan videojuegos especializados para la patología.

La utilización de videojuegos, concretamente en terapia ocupacional, es aún más difícil de encontrar. Algunos ejemplos encontrados son los siguientes:

- El estudio de Cano-Mañas (2017), que aborda la rehabilitación de pacientes con ictus subagudo mediante la utilización de videojuegos, más específicamente mediante la utilización de la Xbox $360^{\circledR}$ y el dispositivo Kinect ${ }^{\circledR}$. Fue realizado por cinco fisioterapeutas y dos terapeutas ocupacionales que, mediante el abordaje rehabilitador, buscaban la mejora del equilibrio, del control postural para evitar caídas y sobre todo para la independencia funcional en actividades de la vida diaria (AVD), además de incrementar la motivación, la autoestima y la adhesión a la intervención. Toda esta intervención se realizó con videojuegos comerciales como el Kinect Sports I ${ }^{\circledR}$, Kinect Sports II ${ }^{\circledR}$, Kinect Joy Ride ${ }^{\circledR}$ y Kinect Adventures ${ }^{\circledR}$.

- El estudio de MG Fernandes (2017), donde se utilizó la videoconsola Wii y el videojuego Wii Fit Plus, en dos casos diagnosticados con la enfermedad de Parkinson. Estos casos presentaron una mejora del equilibrio, sugiriendo el beneficio de la Nintendo Wii en el entrenamiento de equilibrio en individuos con la enfermedad de Parkinson.

- El estudio de Mendelevich (2016), que utilizó el videojuego Wii Fit para determinar la efectividad del uso del videojuego junto con tratamientos convencionales para rehabilitar el equilibrio en pacientes con amputaciones de miembro inferior.

- El estudio de Peñuelas Calvo et al. (2020), argumentó que se podían prescribir sesiones del videojuego "EndeavourRX". Fue creado en particular para el TDAH, para mejorar su atención de los jóvenes. Este juego consiste en pilotar una nave en varios mapas alienígenas, submarinos o volcanes, entre otros.

- El estudio de Walker (2014), que promociona la utilización de videojuegos en realidad virtual como método de intervención en adultos.

- El estudio de Zolyomi (2017), que utiliza el videojuego "Minecraft" como método de intervención para trabajar las habilidades sociales y aplicarlas en un ambiente virtual, interactuando con otros 
jugadores. Presenta un ambiente que lleva a infinidad de cosas que hacer y de interacciones sociales. Además, en este estudio se utiliza el servidor "Autcraft" que es un servidor del videojuego para niño/ as con autismo además de sus familiares.

A través de este estudio se pretende aplicar un nuevo método de intervención para el trastorno por déficit de atención e hiperactividad, mediante el uso de videojuegos comerciales.

\section{Metodología}

El presente estudio es de tipo cuasi-experimental, antesdespués o pre-post. Se realizó una evaluación previa y posterior a la aplicación de la intervención a través de los videojuegos.

Se escogió a usuarios del centro DIVERTEA en Cáceres (España), de una edad comprendida entre 5 y 10 años que presentasen déficit de atención, bien como TDAH o bien como trastorno comórbido de alguna otra patología o trastorno. La muestra final consistió en un grupo de tres niños de edades entre 5 y 7 años del centro DIVERTEA en Cáceres (España) que presentaban déficit de atención. Uno de los niños estaba diagnosticado de TDAH de tipo inatento, mientras que los otros dos niños estaban diagnosticados de Trastorno del Espectro Autista (TEA) pero presentaban déficit de atención.

\section{Materiales}

Se utilizó un videojuego indie conocido como "GRIS". Este videojuego fue creado a finales de 2018 por la compañía española "Nomada Studio". De estilo de plataforma, cuenta con 5 niveles, a cada cual más difícil que el anterior, con cada vez más mecánicas.

Fue escogido frente a otros videojuegos de plataformas debido a ser poco conocido por el público en general y no tener así que recurrir a otros videojuegos más clásicos y conocidos como Super Mario o Sonic. Además, otras características como su sencillez gráfica y mecánica; y su historia emocional fueron tenidas en cuenta para su selección como videojuego en el estudio. Fue instalado en un portátil, al cual se le conectó un recep-


consola.
Para la evaluación de los niño/as, se utilizó el Test de Toulouse (Anexo 1), que consiste en una prueba que mide la atención selectiva y sostenida desde los 6 años hasta los 90. El instrumento consta de 1200 figuras. Estas figuras son una serie de cuadrados con una línea en algún lado o ángulo. Su aplicación se realiza en 10 minutos evaluando así la atención sostenida, obteniendo el Índice Global de Atención y Percepción (IGAP), mediante la siguiente formula (Cid, Manríquez, NahueIñir, \& Colil, 2019):

\section{Aciertos-Errores X 100 Aciertos + Omisiones}

Además, se evaluó a través de la observación el rendimiento del niño/a durante las sesiones. Para ello se creó una guía de observación (Anexo 2), la Guía GAME. Fue creada teniendo en cuenta los principales aspectos evaluables en el desempeño de un usuario a la hora de jugar a videojuegos y consta de dos apartados.

El primer apartado evalúa el manejo con el gamepad o mando, además del nivel de interés en los videojuegos. El segundo apartado evalúa ítems relacionados con el videojuego seleccionado, al final de todos estos, se proporciona una puntuación total, siendo la suma de los puntos de cada ítem. Estos son:

- Tema. Se evalúa el interés en el tipo de juego utilizado.

- Historia. Se evalúa la capacidad de reconocer la historia del juego o si por el contrario juega por jugar. Este ítem solo es evaluado si el videojuego contiene una historia o trama clara.

- Sentimientos. Se evalúan los posibles sentimientos que genera el videojuego en la persona.

- Capacidad de frustración. Se evalúa el nivel de frustración que presenta y la capacidad de buscar una solución por sí mismo.

- Capacidad de abstracción. Se evalúa la capacidad de mantener la atención durante la prueba. Además, se le puntúa del 1 al 10 en su nivel de atención.

- Memoria. Se evalúa la capacidad de recordar lo último que se realizó en el videojuego en la anterior sesión. 
Para obtener el permiso de los padres/madres/tutores legales para la participación del niño/a en el estudio, se creó un documento de consentimiento informado/ autorización. (Anexo 3) Este documento fue entregado en mano a cada padre/madre y se les explicó en detalle en que iba a consistir el estudio, se resolvieron las posibles dudas que pudiesen tenery se les proporcionó un teléfono de contacto para cualquier inconveniente o problema que pudiese haber al respecto. Todos los participantes dieron su visto bueno y firmaron el documento. Además, para mantener la privacidad y anonimato, no se utilizaron nombres ni datos más allá de los necesarios para clasificar a cada uno de los participantes.

\section{Descripción de las sesiones}

Se realizaron un total de 5 sesiones, todos los martes durante 5 semanas, a las cuales asistieron los tres participantes.

En la primera sesión se evaluó el nivel de atención actual a través del Test de Toulouse, el cual se realizó en los 10 primeros minutos y tras su finalización, se realizó la primera toma de contacto con el videojuego GRIS. A medida que iban jugando, se les realizó preguntas sobre cómo se podría estar sintiendo la protagonista, si les gustaba el juego y si querrían volver a jugar otro día. Además, se observó su manejo del gamepad, si presentaban algún tipo de dificultad y su frustración y su manera de resolverlo. Todo ello quedó reflejado en la guía de observación GAME.

Las siguientes sesiones consistieron en la utilización del videojuego GRIS, durante los 30 minutos de duración de la sesión, recordando al participante que debía mantener la atención en la pantalla y pensar muy bien las acciones que iba a realizar para avanzar. Además, se les realizó algunas preguntas durante la sesión relacionadas con el videojuego, y que quedaron reflejadas en la guía GAME.
La última sesión consistió en dos partes, una de utilización del videojuego GRIS y otra de evaluación. Por un lado, se utilizó el videojuego GRIS durante 20 minutos, y al igual que las anteriores sesiones se recordó a los participantes que debían mantener la atención y se les fue ayudando en caso de que no supiesen continuar. Una vez finalizados los 20 minutos, se procedió a evaluar al participante mediante el Test de Toulouse, con una duración de 10 minutos. Tras el final de tiempo, se procedió a la despedida del participante y de los familiares en caso de que estuviesen en el centro.

\section{ReSULTADOS}

\section{Participante N. ${ }^{0} 1$ (Figura 1)}

Niño/a de 5 años, diagnosticado de TDAH de tipo Inatento. En la evaluación previa a la intervención, en el Test de Toulouse, obtuvo una puntuación de IGAP del 43\% de un total de 93 figuras, con 42 aciertos, 51 omisiones y 2 errores.

Durante las sesiones se descubrió que no presentaba gran interés por el videojuego propuesto, debido a que su principal interés eran los videojuegos deportivos.

Se observó que empatizaba con la protagonista y los demás personajes. además, presentaba gran cantidad de frustraciones que no trataba de solucionar de manera calmada, sino que, seguía realizando la misma acción sin pensar detenidamente una solución.

Tras la última sesión, en la evaluación final, se le volvió a aplicar el Test de Toulouse, obteniendo una puntuación de IGAP del $77 \%$ de un total de 89 figuras, con 69 aciertos, 20 omisiones y 1 error. 
FIGURA 1 COMPARATIVA ÍNDICE IGAP Y ACIERTOS-OMISIONES-ERRORES PREVIO Y POSTERIOR A LA INTERVENCIÓN DEL PARTICIPANTE $\mathrm{N}^{\mathrm{O}} .1$

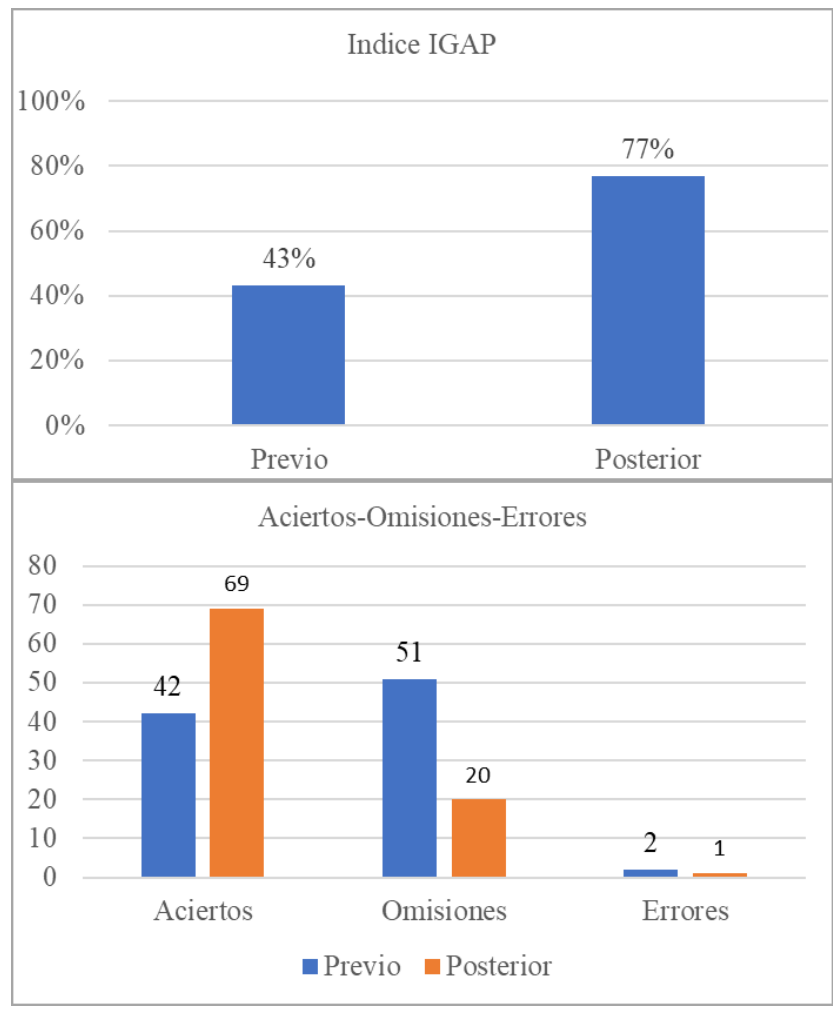

Participante N. ${ }^{\circ} 2$ (Figura 2)

Niño/a de 7 años, diagnosticado de Trastorno del Espectro Autista con déficit de atención. En la evaluación previa a la intervención, en el Test de Toulouse, obtuvo una puntuación de IGAP del $56 \%$ de un total de 102 figuras, con 60 aciertos, 42 omisiones y 2 errores.

Durante las sesiones se descubrió que presentaba un buen manejo del gamepad, presentó gran interés por el videojuego planteado y fue capaz de empatizar con la protagonista y los demás personajes.

No presentó frustraciones y si presentó alguna, intentó solucionarlo de manera calmada. Durante las sesiones, mantuvo correctamente la atención en la pantalla, aunque si alguien se encontraba en la sala perdía parcialmente la atención.
Tras la última sesión, en la evaluación final, se le volvió a aplicar el Test de Toulouse, obteniendo una puntuación de IGAP del $83 \%$ de un total de 77 figuras, con 65 aciertos, 12 omisiones y 1 error.

\section{FIGURA 2 COMPARATIVA ÍNDICE IGAP Y ACIERTOS-OMI- SIONES-ERRORES PREVIO Y POSTERIOR A LA INTERVEN- CIÓN DEL PARTICIPANTE $\mathrm{N}^{0} .2$}

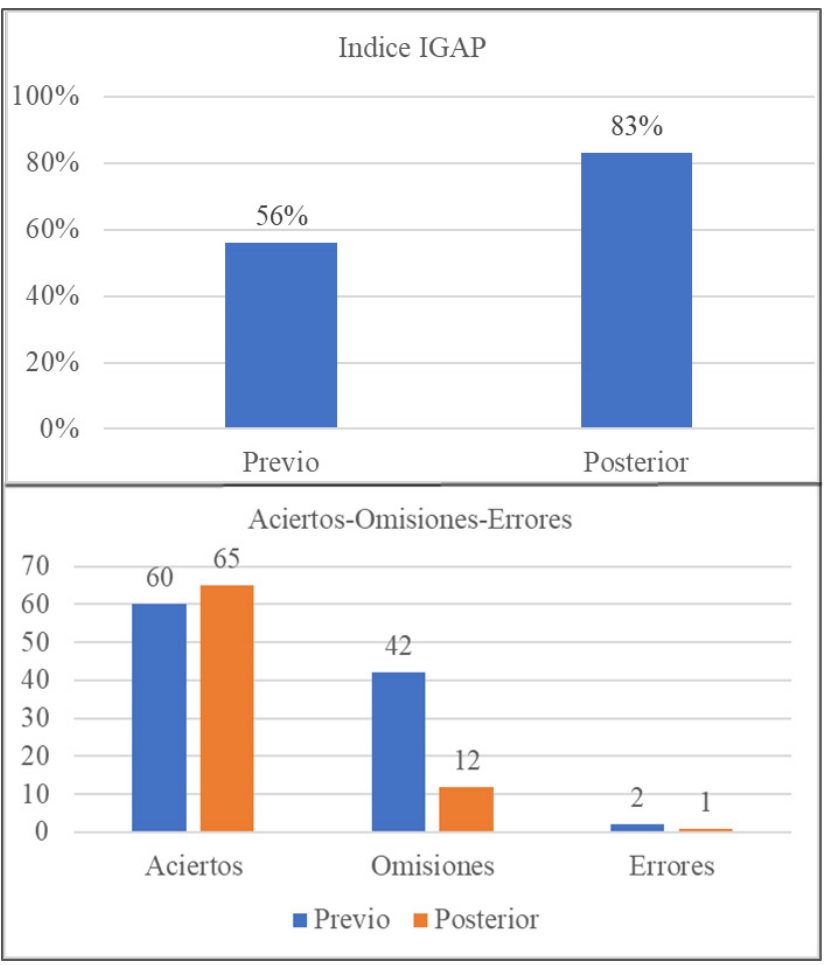

\section{Participante N. ${ }^{\circ} 3$ (Figura 3)}

Niño/a de 7 años, diagnosticado de Trastorno del Espectro Autista con déficit de atención. En la evaluación previa a la intervención, en el Test de Toulouse, obtuvo una puntuación de IGAP del $77 \%$, con 130 aciertos, 34 omisiones y 3 errores.

Durante las sesiones se descubrió que presentaba un extraño manejo del gamepad, con una mala colocación de los dedos, lo que le provocaba cansancio en los mismos al finalizar la sesión.

Se observó que mostraba interés por el videojuego planteado y que una de sus aficiones era jugar a videojuegos. Además, fue capaz de empatizar con la protagonista y 
los diferentes personajes. No presentó grandes frustraciones, y si las presentó, las intentó solucionar. En caso de no poder, pedía ayuda.

Tras la última sesión, en la evaluación final, se le volvió a aplicar el Test de Toulouse, obteniendo una puntuación de IGAP del 97\% de un total de 135 figuras, con 131 aciertos, 4 omisiones y sin errores.

\section{FIGURA 3: COMPARATIVA ÍNDICE IGAP Y ACIERTOS-OMI- SIONES-ERRORES PREVIO Y POSTERIOR A LA INTERVEN- CIÓN DEL PARTICIPANTE $\mathrm{N}^{\mathrm{O}} \cdot 3$}

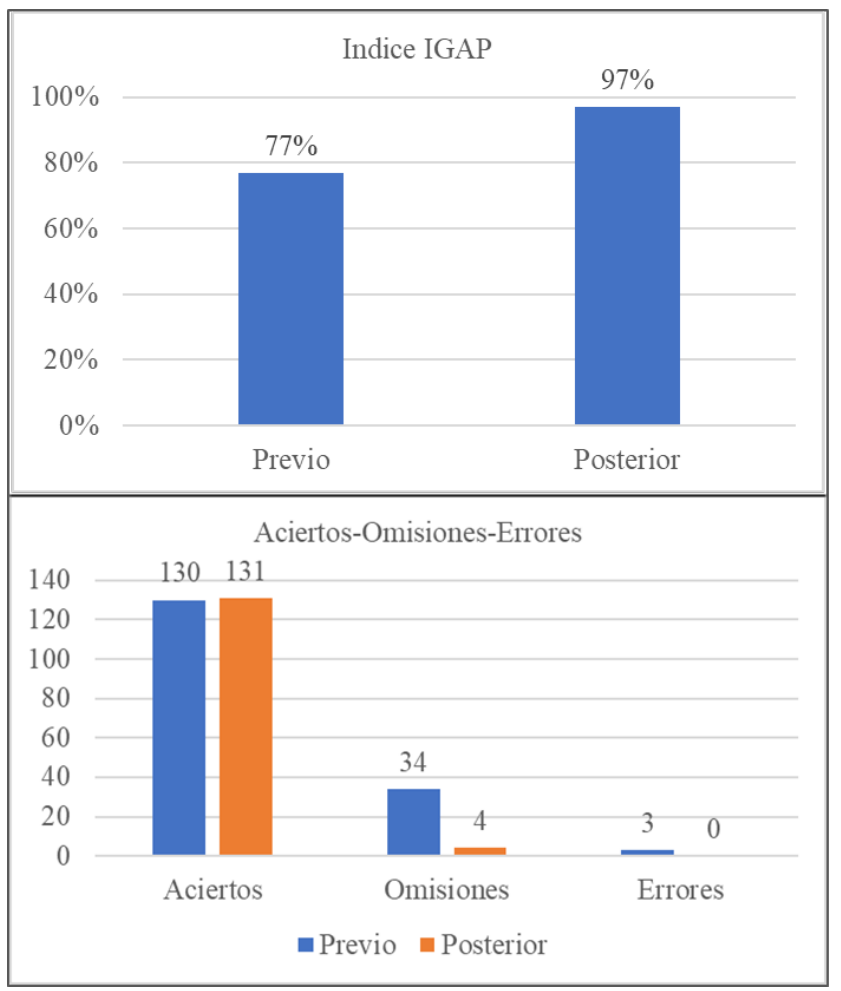

\section{DisCusıón}

El objetivo de este estudio fue aplicar un nuevo método de intervención para diferentes patologías y trastornos, a través de los videojuegos comerciales. Fue planteado debido a que, actualmente, no hay demasiados estudios que apliquen métodos de intervención a través de los videojuegos, y muchos de los que puedan aparecer en las diferentes bases de datos aplican videojuegos creados específicamente para las patologías en cuestión. Como es el caso del estudio de Peñuelas Calvo et al. (2O2O), donde analizaron un videojuego creado en especial para el TDAH.

Nuestro estudio considera que, para el paciente no es de gran interés jugar a videojuegos específicos para la patología, ya que normalmente, suelen ser de corta duración, repetitivos y de baja calidad. Los participantes tendrán mayor interés en las sesiones si el videojuego utilizado es algo que le llame la atención y le atraiga, tanto por las mecánicas como por la historia. Por ello, sería interesante analizar los diferentes videojuegos comerciales y ver que beneficios pueden ofrecer como método de intervención para todo tipo de patologías.

Los resultados obtenidos con este estudio muestran los beneficios de los videojuegos como método de intervención en niños/as con TDAH o con déficit de atención como trastorno comórbido. Compartiendo similitudes con los resultados obtenidos por otros investigadores, que obtuvieron beneficios mediante la utilización de videojuegos, como Minecraft (Zolyomi \& Schmalz, 2017), Wii Fit Plus (Fernandes et al., 2017) o Kinect Sports I \& II (Cano-Mañas et al., 2017), para diferentes tipos de patologías y trastornos.

Respecto a las limitaciones, este estudio presenta un número de participantes reducido, con tan solo 3 participantes. En otros estudios, el número de participantes es mucho mayor, como es el caso del estudio de CanoMañas (2017) que tuvo un total de 21 participantes, el estudio de Mendelevich (2016) que tuvo un total de 14 participantes y el estudio de Zolyomi (2016) que tuvo un total de 6 participantes. Otra limitación fue el número de sesiones realizadas, un total de 5 sesiones durante 5 semanas, es decir, una sesión por semana. Este número es muy reducido en comparación con otros estudios. En el estudio de Mendelevich et al. (2016) se realizaron 14 sesiones, 2 sesiones durante 7 semanas. En el estudio de Cano-Mañas (2017) se realizaron sesiones a lo largo de un total de 8 semanas. Y en el estudio de Fernandes (2017) se realizaron un total de 10 sesiones, dos veces por semana durante 1 mes.

Este estudio comparte la idea de que los videojuegos pueden ser utilizados como método de intervención (Cano-Mañas et al., 2017; Fernandes et al., 2017; Zolyomi \& Schmalz, 2017) y como herramienta fundamental para el desarrollo de habilidades (de Prado, 2018; Restrepo Escobar, Taborda, Magdaly, \& Arboleda Sierra, 2019). 
Cabe destacar que, durante la realización de las sesiones, aparecieron diversos problemas con el ordenador portátil, pero aun así se buscó la manera de mantener el interés del participante hasta que el problema quedó solucionado.

\section{CONCLUSIONES}

Con los resultados obtenidos, podemos observar una mejora en los niveles de atención de los tres participantes y una reducción de omisiones y fallos en el Test de Toulouse en comparación con los datos previos a la intervención. Esta reducción de omisiones y fallos nos indica que los participantes prestaron mayor atención a cada figura del test.

Los datos nos confirman que los videojuegos son un buen método de intervención para el trastorno por déficit de atención e hiperactividad, abriendo la puerta a aplicar este método en otros tipos de patologías y trastornos en muestras mayores de participantes.

\section{AgRADECIMIENTOS}

Agradecer a DIVERTEA, a su directora y a los profesionales permitirnos realizar el estudio en su centro, a los participantes y a los padres de los participantes por acceder a realizarlo.

\section{REFERENCIAS BiLBIOGRÁFICAS}

American Psychiatric Association. (2013). Diagnostic and statistical manual of mental disorders (DSM-5 $\left.{ }^{\circledR}\right)$ American Psychiatric Pub.

Artigas-Pallarés, J., Nonell, K. G., \& Ratera, E. R. (2013). Comorbilidad en el TDAH.

Cambrige Dictionary. (2017). Cambridge dictionary. Avaliable at: Http:// Dictionary.Cambridge.Org,

Cano-Mañas, M. J., Collado-Vázquez, S., \& Cano-de-la-Cuerda, R. (2017). Videojuegos comerciales en la rehabilitación de pacientes con ictus subagudo: Estudio piloto. Revista De Neurología, 65(8), 337-347.

Cid, F. M., Manríquez, A. B., Nahuelñir, M. A. R., \& Colil, D. F. (2019). Valores normativos del test de toulouse-piéron de atención selectiva y sostenida en una muestra de estudiantes de educación física de santiago de chile. Revista Ciencias De La Actividad Física, 20(1), 1. Retrieved from https://dialnet.unirioja.es/servlet/ articulo? codigo $=6844664$ de Prado, M. G. (2018). Beneficios educativos y videojuegos: Revisión de la literatura española. Education in the Knowledge Society, 19(3), 37-51.

del Río, J. E. (2014). Trastorno por déficit de atención e hiperactividad (TDAH). Cuadernos Del Tomás, (6), 117-130.

Fernandes, M. G., Silva, R. B., da Rosa, C. M., Almeida, S., de Lima, Ana Karolina Pontes, de Arruda, G. T., \& Braz, M. M. (2017). A influência do nintendo WII no equilíbrio de indivíduos com doença de parkinson: Relato de casos. Revista Kairós: Gerontologia, 20(4), 403-413.

Fontiveros, M., a Ángeles, M., Vera, M., a José, M., Tejada González, A., González Igeño, V., \& García Resa, O. (2015). Actualización en el tratamiento del trastorno del déficit de atención con/sin hiperactividad (TDAH) en atención primaria. Revista Clínica De Medicina De Familia, 8(3), 231-239.

GameTrack digest: Quarter 3. (2018). Online], Interactive Software Federation of Europe (ISFE), Https://Www.Isfe.Eu/Sites/Isfe.Eu/Files/ gametrack_european_summary_data_2018_q3.Pdf, , 1 .

Gómez, G. C. (2017). Ventajas y desventajas del uso del videojuego como herramienta didáctica en las ciencias sociales.

Martín, M del Mar Badia, Muntada, M. C., Busquets, C. G., Pros, R. C., \& Sáez, T. D. (2015). Videojuegos, televisión y rendimiento académico en alumnos de primaria. Pixel-Bit.Revista De Medios YEducación, (46), 25-38.

Mendelevich, A., Módica, M., Kramer, M., Gallo, S., \& Ostolaza, M. (2016). Efectividad de la nintendo wii fit ${ }^{\circledR}$ en la rehabilitación del equilibrio en sujetos con amputación unilateral de miembro inferior. Boletín Del Departamento De Docencia E Investigación IREP, 15(1)

PeñuelasCalvo, I., JiangLin, L. K., GirelaSerrano, B., DelgadoGomez, D., Navarro-Jimenez, R., Baca-Garcia, E., \& Porras-Segovia, A. (2020). Video games for the assessment and treatment of attentiondeficit/hyperactivity disorder: A systematic review. European Child \& Adolescent Psychiatry,

Pérez, M. R. (2015). Tratamiento cognitivo-conductual de conductas disruptivas en un niño con TDAH y trastorno negativista desafiante. Revista De Psicología Clínica Con Niños YAdolescentes, 2(1), 45-54.

Restrepo Escobar, S. M., Taborda, A., Magdaly, L., \& Arboleda Sierra, W. (2019). El rendimiento escolar y el uso de videojuegos en estudiantes de básica secundaria del municipio de la estrellaantioquia. Revista Educación, 43(2), 122-134.

Rodillo, B. E. (2015). Trastorno por déficit de atención e hiperactividad (TDAH) en adolescentes. Revista Médica Clínica Las Condes, 26(1), 52-59.

Rodríguez Molinero, L., López Villalobos, J. A., Garrido Redondo, M., Sacristán Martín, A. M., Martínez Rivera, M. T., \& Ruiz Sanz, F. (2009). Estudio psicométrico-clínico de prevalencia y comorbilidad del trastorno por déficit de atención con hiperactividad en castilla y león (españa). Pediatría Atención Primaria, 17(42), 251-270. 
Rosa Cortés, S. (2015). Diseño ergonómico de un controlador de videojuegos profesional.

Rubiales, J., Bakker, L., Russo, D., \& González, R. (2016). Desempeño en funciones ejecutivas y síntomas comórbidos asociados en niños con trastorno por déficit de atención con hiperactividad (TDAH). CES Psicología, 9(2), 99-113.

Sánchez, G. F. L., Sánchez, L. L., \& Suárez, A. D. (2015). Trastorno por déficit de atención con hiperactividad (TDAH) y actividad física. EmásF: Revista Digital De Educación Física, (32), 53-65.

Taniel, M., \& Samuel, P. P. (2015). Diseño de controladorytemporizador automático de computadores y consolas de videojuego.
Valderrama-Ramos, J. A. (2011). Videojuegos y educación: Explorando aprendizajes entre adolescentes.

Walker, B. A. (2014). The acceptance and use of virtual gaming as an intervention strategy for older adults in occupational therapy. Games for HealthJournal, 3(6), 333-340. doi:10.1089/g4h.2014.0062

Zolyomi, A., \& Schmalz, M. (2017). Mining for social skills: Minecraft in home and therapy for neurodiverse youth.

Zúñiga, A. H., \& Forteza, O. D. (2014). El TDAH y su comorbilidad. Pediatría Integral, 18(9), 643-654.

TEST DE TOULOUSE

\section{Anexo 1: Test de Toulouse}

\section{ANEXOS}

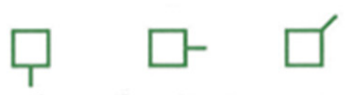

๙

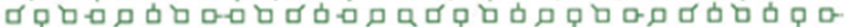

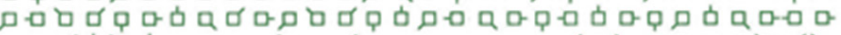

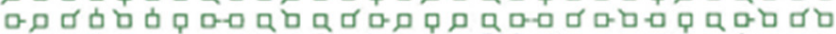



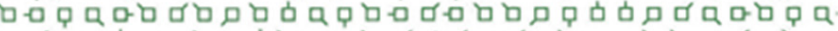

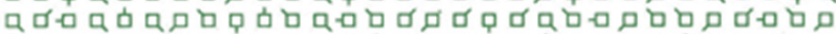

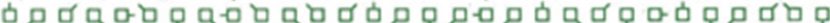
ص

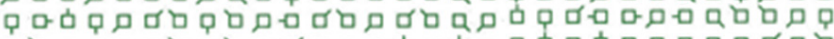

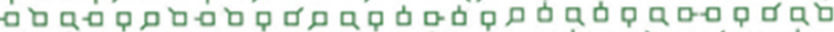

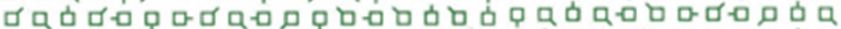

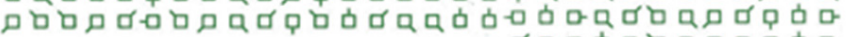



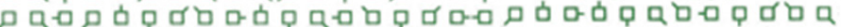



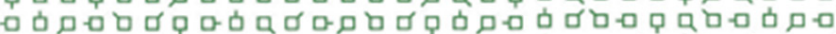

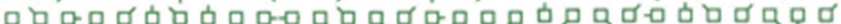

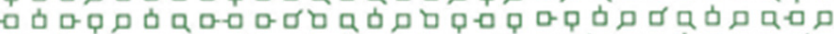

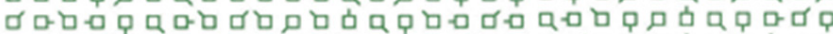

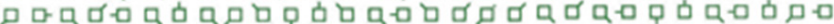

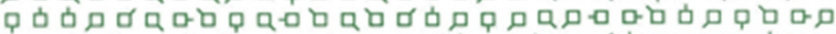

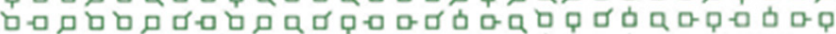



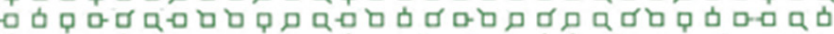

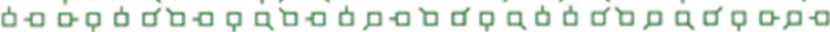

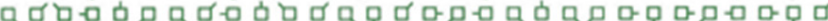

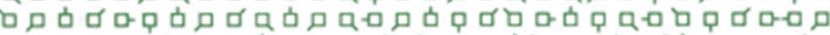

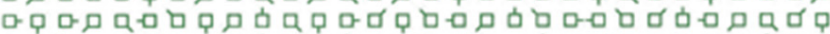

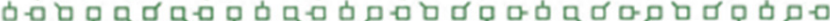

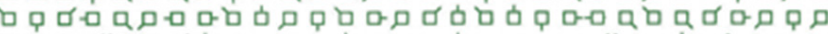

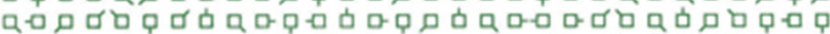

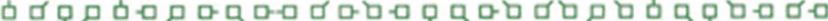
๙

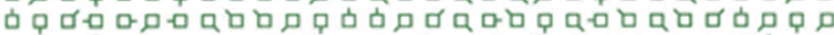

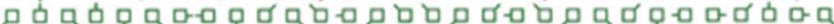





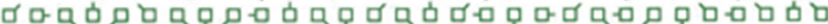

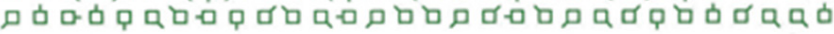




\section{Anexo 2: Guía de Observación GAME}

\begin{tabular}{|c|c|}
\hline Nombre y Apellidos & \\
\hline \multicolumn{2}{|l|}{ Edad } \\
\hline \multicolumn{2}{|l|}{ Sexo } \\
\hline \multicolumn{2}{|l|}{ Fecha } \\
\hline \multicolumn{2}{|r|}{ Ítems Generales } \\
\hline Gamepad & $\begin{array}{l}\text { 1. Lo conoce, pero no lo sabe utilizar. } \\
\text { 2. Lo utiliza con dificultad. } \\
\text { 3. Presenta pequeños problemas en la utilización. } \\
\text { 4. Lo utiliza con total normalidad. }\end{array}$ \\
\hline Videojuegos & $\begin{array}{l}\text { 1. Juega siempre a los mismos juegos y no está abierto a nuevos } \\
\text { tipos. } \\
\text { 2. Juega siempre a los mismos juegos y está abierto a nuevos tipos. } \\
\text { 3. Presenta una mayor variedad de juegos (al menos } 3 \text { o } 4 \text { de dife- } \\
\text { rentes temáticas). } \\
\text { 4. Juega a cualquier juego que se le proponga. }\end{array}$ \\
\hline \multicolumn{2}{|r|}{ Ítems Específicos } \\
\hline Tema & $\begin{array}{l}\text { 1. No conoce el tema del juego (Juega por jugar). } \\
\text { 2. Conoce el tema del juego. }\end{array}$ \\
\hline $\begin{array}{c}\text { Historia } \\
\text { (Si el juego no tiene historia no se } \\
\text { evalúa) }\end{array}$ & $\begin{array}{l}\text { 1. No reconoce la historia del juego. } \\
\text { 2. Reconoce la historia del juego. }\end{array}$ \\
\hline Sentimientos & $\begin{array}{l}\text { 1. No le provoca ningún tipo de sentimiento. } \\
\text { 2. Empatiza con el protagonista. }\end{array}$ \\
\hline Capacidad de Frustración & $\begin{array}{l}\text { 1. Se frustra y se bloquea. } \\
\text { 2. Se frustra, no se bloquea y busca una solución. }\end{array}$ \\
\hline Capacidad de Abstracción & $\begin{array}{l}\text { 1. No es capaz de mantener la atención. } \\
\text { 2. Mantiene la atención durante gran parte de la sesión. }\end{array}$ \\
\hline $\begin{array}{l}\text { Memoria } \\
\text { (Evaluar a partir de la primera sesión) }\end{array}$ & $\begin{array}{l}\text { 1. No recuerda lo que tenía que hacer o por donde iba. } \\
\text { 2. Recuerda las acciones y por donde iba. }\end{array}$ \\
\hline Puntuación Total & \\
\hline
\end{tabular}


Dificultades

(Describir las dificultades que ha tenido)

Nivel de Atención

(1 al 10)

Anexo 3: Consentimiento Informado/ Autorización

\section{CONSENTIMIENTO INFORMADO/AUTORIZACIÓN}

“Gaming como método de intervención en el trastorno por déficit de atención e hiperactividad"

Dn/ña: , mayor de edad, con DNI

\section{MANIFIESTO:}

Que he sido informado de los fines y objetivos del Trabajo Fin de Grado "Gaming como método de intervención en el trastorno por déficit de atención e hiperactividad", para la consecución del Grado de Terapia Ocupacional en la Universidad de Extremadura, realizado por JORGE CASADO GARCíA con el DNI 76oXXXXXX y AUTORIZO a mi hijo/a, para que participe, de forma voluntaria en la intervención.

Comprendo y estoy de acuerdo con que, para la realización del trabajo, se tomarán datos de manera ANÓNIMA, antes y después de la intervención.

Esta intervención no tendrá ningún tipo de coste ni riesgo para el participante, y podrá retirarse de la misma cuando quiera. Sin necesidad de dar explicaciones, si así lo considera.

Cáceres, a de de

Fdo.: Jorge Casado García

DNI: 760XXXXXX

Estudiante de $4^{\circ}$ Curso Grado en Terapia Ocupacional
Fdo:

Padre/madre, del participante. 